Meta

Journal des traducteurs

Translators' Journal

\title{
Aspects pédagogiques de l'évaluation des traductions
}

\section{Hannelore Lee-Jahnke}

Volume 46, numéro 2, juin 2001

Évaluation : paramètres, méthodes, aspects pédagogiques /

Evaluation: Parameters, Methods, Pedagogical Aspects

URI : https://id.erudit.org/iderudit/003447ar

DOI : https://doi.org/10.7202/003447ar

Aller au sommaire du numéro

Éditeur(s)

Les Presses de l'Université de Montréal

ISSN

0026-0452 (imprimé)

1492-1421 (numérique)

Découvrir la revue

Citer cet article

Lee-Jahnke, H. (2001). Aspects pédagogiques de l'évaluation des traductions. Meta, 46(2), 258-271. https://doi.org/10.7202/003447ar

\section{Résumé de l'article}

Partant de l'adage pédagogique qu'on ne saurait bien faire que ce dont on comprend parfaitement l'objectif, notre propos est de montrer des approches novatrices dans les trois domaines suivants : 1 . Différentes méthodes pour sensibiliser les étudiants à l'évaluation en général ; 2. L'évaluation " formative » telle qu'elle est pratiquée dans nos cours ; 3 . Projet sur une évaluation "sommative". d'utilisation que vous pouvez consulter en ligne.

https://apropos.erudit.org/fr/usagers/politique-dutilisation/ 


\title{
Aspects pédagogiques de l'évaluation en traduction
}

\author{
HANNELORE LEE-JAHNKE \\ Université de Genève, Genève, Suisse
}

\section{RÉSUMÉ}

Partant de l'adage pédagogique qu'on ne saurait bien faire que ce dont on comprend parfaitement l'objectif, notre propos est de montrer des approches novatrices dans les trois domaines suivants:

1. Différentes méthodes pour sensibiliser les étudiants à l'évaluation en général;

2. L'évaluation «formative» telle qu'elle est pratiquée dans nos cours;

3. Projet sur une évaluation «sommative».

\section{ABSTRACT}

It is pedagogically axiomatic that true quality can only be achieved if the goal is made perfectly clear to the student. This paper discusses a fresh approach to the following issues:

1. Various methods to highten awareness of students perception of evaluation;

2. formative evaluation as used in our courses;

3. Ongoing research in sommative evaluation.

\section{MOTS-CLÉS/KEYWORDS}

évalutation en traduction, aspects pédagogiques, évaluation formative, évaluation sommative, processus cognitifs

\section{Introduction}

Depuis le Siècle des lumières, la pédagogie a été un paradis pour les philosophes aux intentions généreuses. Cela explique, en partie du moins, pourquoi elle s'est transformée en un temple dédié à la créativité et à l'évaluation. Si l'on admet qu'il est impossible d'enseigner la créativité tandis qu'il est possible de l'apprendre et d'apprendre également les principes fondamentaux de l'évaluation, les pédagogues célèbrent volontiers cet esprit antihiérarchique qui fait de la créativité un emblème d'égalité (Schmidt 1988: 37). Dès lors, ils se limitent, dans leur pratique professionnelle, à initier ou à éveiller le don créateur de leurs disciples. Inversement, il est considéré comme antipédagogique de transmettre de lourdes quantités de savoir ou d'adopter des méthodes éducatives par trop rigides. Qu'en est-il dans ces conditions de l'approche pédagogique en évaluation?

Depuis les travaux de Binet ${ }^{1}$ au début du siècle, les psychologues pensent être en mesure d'améliorer l'évaluation. De fait, la docimologie d'un côté, les tests de connaissance d'un autre, ont été développés entre les deux guerres, parallèlement à la psychologie différentielle. Bien plus, la psychologie générale a contribué à mieux comprendre le comportement de jugement des notateurs, comme l'ont montré les travaux de Noizet et Caverni (1978), ou ceux de Bonniol (1981). Les exemples de pédagogie donnés par Bloom (1972) qui supposent un apprentissage cumulatif mais 
linéaire, et au cours duquel l'étudiant peut progresser régulièrement, représentent indubitablement un modèle d'évaluation. Un modèle que bien des professeurs s'approprient.

Une épreuve pédagogique devrait normalement rendre compte des apprentissages réalisés, même s'ils ne différencient plus du tout les étudiants, du fait qu'ils sont acquis de façon générale. C'est pourquoi des recherches restent nécessaires pour fonder une évaluation pédagogique qui puisse mieux répondre aux besoins de l'ensemble des étudiants. Ce genre de recherches doit obligatoirement mettre à profit toutes les connaissances qui ont été faites dans le domaine de la créativité en pédagogie (Piaget 1974).

Nous avons essayé et essayons, dans nos cours, d'appliquer des principes pédagogiques qui incitent à la créativité.

L'évaluation en traduction est un thème à la mode au point même de susciter actuellement des enquêtes internationales via Internet ${ }^{2}$ (voir annexe I), de nombreux colloques et des articles de revues spécialisées (The Translator 2000). Pourquoi? Parce que, d'une part, les critères d'évaluation ne sont pas aussi clairs qu'on le souhaiterait et que, d'autre part, notre profession, dont la notoriété s'accroît, est en quête d'une assise forte en ce domaine. En effet, la traductologie ne peut être respectée à sa juste valeur que si elle est en mesure de fournir des «produits» de qualité. Une traduction de qualité exige donc, comme tout autre produit, qu'un certain nombre de conditions précises soient préalablement remplies. L'une d'elle se résume ainsi: l'évaluation est à mettre en œuvre dès le début de l'enseignement. C'est la raison pour laquelle nous attachons une grande importance à cette démarche préliminaire en faisant nettement la distinction entre a) l'évaluation formative, et b) l'évaluation «sommative». La première ayant, à nos yeux, une importance majeure pour les raisons pédagogiques évoquées plus haut.

Pourtant, quand on pense "évaluation», on désigne en général l'évaluation dite "sommative», c'est-à-dire celle qui sanctionne, et qui, dans la plupart des cas, s'exprime en chiffres, en notes «facilement» attribuées. En réalité, les méthodes et modes de calcul posent d'autres problèmes et maintes tentatives ont été faites, et vont encore être entreprises, afin de rendre cet exercice aussi fiable et objectif que possible. Le présent numéro de Meta nous en propose d'ailleurs quelques-unes, notamment les travaux de recherche de Waddington (2001) et Williams (2001), pour n'en citer que deux.

Comme les méthodes d'évaluation se multiplient depuis des années et que tout un chacun essaie de trouver une voie aussi objective que possible, une deuxième question surgit tout naturellement: pourquoi cet état de fait? Bien évidemment, nous ne nous situons pas dans les sciences exactes où une notation serait relativement «facile», car objectivable. Nous traitons avec une matière qui est délicate à schématiser, en raison de la subjectivité des critères. Il est vrai qu'une terminologie unique, comprise par tous, fait encore défaut dans bien des langues et, même, dans les langues où ce «métalangage» existe, celui-ci est ignoré par bon nombre d'enseignants.

Notre domaine souffre donc encore d'un manque d'affirmation, d'un manque de structure. La description de l'historien (Delisle 2001), qui ouvre le présent numéro, nous l'a excellemment décrit. Cette carence se fait d'autant plus sentir que les universités, chargées de la formation des étudiants en traductologie, veulent donner à cet enseignement, à visée essentiellement pratique, une assise scientifique ${ }^{3}$. 
Scientifique, qu'est-ce que cela veut dire en traduction? Cela veut dire que les exercices proposés puissent être reproductibles au même titre que les expériences en laboratoire scientifique. Est-ce réalisable? D'éminents chercheurs ont travaillé dans ce domaine (Gerzymisch-Arbogast 1998) et ont publié des travaux remarquables. La tâche est complexe, car il faut, en corrigeant les écrits des étudiants, pondérer les erreurs d'après leur gravité. Cette pondération soulève une autre problématique qui a été abordée avec beaucoup de perspicacité par Peter Axel Schmitt (2001).

Une telle question s'est posée à nous lors d'un examen final. Dans le travail fourni - excellent dans sa globalité — un mot-clé du texte avait été confondu ${ }^{4}$. S'il ne s'était agi que d'une faute de vocabulaire, l'appréciation de l'ensemble du texte aurait été positive. Toutefois, comme cette confusion ponctuelle enlevait toute signification pratique au document, nous avons opté pour une note insuffisante. Par cette manière de faire, il nous semble utile de présenter les premiers éléments de notre système de notation et du même coup nous posons la question: quel est le rôle de l'évaluation dans l'enseignement?

En effet, tous les professeurs n'ont pas la même conception du rôle de l'évaluation. Pour beaucoup, elle sert uniquement à sanctionner les travaux à la fin du cours. Pour d'autres, par contre, elle sert à aider les étudiants à s'améliorer dans leurs performances. À notre avis, cette amélioration ne vise pas seulement la réussite dans les examens. Elle doit essentiellement viser l'amélioration de l'aptitude à traduire et, ensuite seulement, le produit fini, à savoir: une traduction de qualité.

C'est dire que l'évaluation formative nous intéresse en priorité ${ }^{5}$. Abordons ce premier volet.

\section{Méthodes pour sensibiliser les étudiants à l'évaluation}

Pourquoi faut-il sensibiliser les étudiants à l'évaluation? Parce que l'évaluation constitue, à nos yeux, l'outil par excellence de tout processus d'apprentissage. Aussi convient-il de la transmettre aux étudiants dans son ampleur afin que ceux-ci l'utilisent pour le mieux.

Pour l'étudiant, l'information nouvelle qu'il reçoit sur un domaine de connaissance reste d'abord indifférenciée. C'est l'exercice, introduit à propos de ce savoir théorique, qui va donner un sens à ces notions, leur apporter du relief et mettre en évidence des aspects plus prégnants, cela sur un fond de connaissances passives. Ses erreurs lui apportent ensuite des informations critiques qui l'obligent à rectifier sa conception préalable, à différencier sa perception dans une région où sa représentation était jusque-là relativement confuse. Parallèlement, pour le professeur, la découverte des tâtonnements de ses étudiants est une occasion de mieux appréhender la structure de la discipline qu'il enseigne. Très souvent, en effet, ce sont les mêmes obstacles épistémiques qui ont marqué l'histoire d'une science, bloquant pendant un temps son progrès et qui font aussi difficulté pour les étudiants.

En examinant les erreurs des étudiants, le professeur est à même de découvrir leurs représentations et sur quels points elles sont erronées. Il peut aussi discerner la source de leur difficulté, les raisons d'un mauvais décodage qui peut être dû à une formulation ambiguë, mais également à des problèmes plus larges, par exemple à la présentation qu'il a donnée de ses exigences et de ses critères d'évaluation (LeeJahnke 2001). 
La deuxième question qui s'enchaîne tout naturellement est alors: comment sensibiliser? En tant que professeur, il importe d'être convaincu de la nécessité de l'évaluation comme instrument de travail pour aboutir à une bonne qualité de traduction. Cela en constitue la conditio sine qua non. Les étapes pédagogiques à suivre consistent, en premier lieu, à créer une atmosphère de confiance afin de favoriser une critique constructive; toute critique se révèle ainsi ressentie comme une facteur positif d'amélioration. En outre, il sera demandé au professeur «idéal» d'avoir quelques connaissances dans le domaine des processus cognitifs, voire même de la psychologie cognitive.

Cette science, on le sait, a un objectif majeur, celui de retracer les processus internes qui jouent un rôle dans les performances cognitives ou mentales. Même avec un but clairement défini, le cheminement pour l'atteindre est encore sujet à controverse. Nous optons pour une approche qui est à même de prendre en compte les informations reçues par le cerveau (Lachmann et Butterfield 1979). Dans le traitement de ces informations, les éléments suivants entrent en jeu:

L'attention;

La perception;

Le processus cognitif.

- Le concept «d'attention» a reçu tellement de significations différentes que certains psychologues sont d'avis qu'il est trop vague et ne devrait pas trouver sa place dans les sciences. Parfois il est employé comme synonyme de concentration et, d'autres fois, on l'utilise comme une capacité de choisir une stimulation venant de l'extérieur afin de procéder à son analyse. Généralement, le concept d'attention est utilisé en tant que sélectivité dans un processus. L'éminent psychologue américain William James (1890: 403-404) l'emploie dans ce sens:

Everyone knows what attention is. It is the taking possession by the mind, in clear and vivid form, of one out of what seem several simultaneously possible objects or trains of thought. Focalisation, concentration, of consciousness are of its essence. It implies withdrawal from some things in order to deal effectively with others.

Les travaux de recherche respectifs à l'attention font la distinction entre "l'attention sélective ${ }^{6} »$ et "l'attention divisée $e^{7} »$. Nous devons, en pédagogie, maintenir cette distinction et l'exercer avec les étudiants en développant des exercices appropriés.

- La perception, le second élément, a été clairement défini par Levine et Schefner (1981): "Perception refers to the way in which we interpret the information gathered (and processed) by the senses. In a word, we sense the presence of a stimulus, but we perceive what it is ». Ces définitions devraient nous guider dans nos approches pédagogiques.

- Le troisième élément, le processus cognitif, nous paraît extrêmement important en pédagogie. Pour satisfaire aux exigences de ce processus et pour illustrer le fonctionnement cognitif de la lecture, toujours dans l'objectif d'une évaluation formative, nous employons le modèle suivant: 


\begin{tabular}{|l|l|l|}
\hline Lecteur & Texte & Contexte \\
\hline $\begin{array}{l}\text { Structures } \\
\text { Connaissances }\end{array}$ & $\begin{array}{l}\text { Intention de l'auteur } \\
\text { Structure du texte }\end{array}$ & $\begin{array}{l}\text { Psychologique } \\
\text { Intention du lecteur } \\
\text { Intérêt }\end{array}$ \\
\hline $\begin{array}{l}\text { Processus de lecture } \\
\text { Capacités }\end{array}$ & $\begin{array}{l}\text { Contenu } \\
\text { Concepts }\end{array}$ & $\begin{array}{l}\text { Social } \\
\text { Intervention du professeur } \\
\text { Intervention de l'étudiant }\end{array}$ \\
\hline & & $\begin{array}{l}\text { Physique } \\
\text { Temps } \\
\text { Bruit }\end{array}$ \\
\hline & & \multicolumn{2}{|l}{} \\
\hline
\end{tabular}

Ensuite nous avons appliqué dans nos cours - et avec un certain succès - différentes approches, et notamment celle décrite ci-après:

\section{a. L'autoévaluation}

Depuis les premiers travaux de Bloom (1972), centrés principalement sur les différentes méthodes pour améliorer l'évaluation des connaissances des étudiants, notre préoccupation a porté sur l'évaluation des connaissances et sur la connaissance de l'évaluation. Par connaître l'évaluation, il convient d'entendre l'approfondissement du rôle joué par l'évaluation comme instrument de régulation des apprentissages des étudiants.

L'autoévaluation $^{8}$ apparaît ainsi comme un moyen d'assurer une régulation continue des apprentissages, et cela de façon individuelle. Le professeur ne peut suivre individuellement chaque étudiant et même, s'il en était capable, nous savons maintenant que ce ne serait pas nécessairement la meilleure chose pour le développement de l'autonomie et de la responsabilité des étudiants. L'auto-évaluation assure donc:

une continuité des régulations qui permet à l'étudiant d'exercer une forme de contrôle cognitif sur tous les aspects de l'opération traduisante;

une différenciation des régulations qui respecte le principe des besoins propres de chaque étudiant.

De nombreuses recherches ont démontré le rôle efficace de l'autoévaluation dans l'apprentissage (Guignard 1998), même s'il est vrai que ce champ d'étude était à peine envisagé en pédagogie voilà une vingtaine d'années. En ce qui concerne la traduction, les recherches sont assurément plus récentes (Lee-Jahnke 1998). Dès lors, il semblerait qu'en poursuivant nos efforts de recherche dans cette voie nous en viendrions à créer des situations didactiques et d'évaluation de manière à «optimiser» l'apprentissage. Cependant, avant que cela ne soit possible, il importe d'intégrer un facteur d'apprentissage déterminant: la motivation d'accomplissement.

Pour que l'autoévaluation soit possible, pour qu'il y ait intervention de stratégies métacognitives, l'engagement cognitif de l'étudiant s'impose. En effet, l'étudiant n'est pas susceptible de surveiller ses processus d'apprentissage si la tâche ne l'intéresse pas et s'il perçoit qu'il lui est impossible d'atteindre l'objectif visé. En fait, lorsque l'étudiant met en jeu des stratégies métacognitives, cette démarche prouve qu'il est déjà motivé. Voilà notre point de départ pour l'exercice proposé ci-après. 
Dès le début de leurs études, les étudiants sont encouragés à mentionner, en fin de document, non seulement les ressources documentaires utilisées pour le travail en question, mais aussi à énumérer les difficultés rencontrées durant leur travail. Pour ce faire, nous leur laissons, d'entrée de jeu, un libre choix de procédure. Autrement dit, les difficultés possibles ne sont pas indiquées et l'expérience montre qu'une telle approche «libre» et «individuelle», voire «individualiste» en début de parcours, met à l'aise. Après plusieurs semaines d'exercice, elle permet à l'élément créateur si stimulant en pédagogie - de jouer tout son rôle.

La deuxième phase de cette façon de sensibiliser à l'évaluation porte sur la structuration des difficultés recensées. Cette phase doit nécessairement être travaillée en groupe et il s'est avéré judicieux de la faire diriger par l'un des étudiants. Durant cette étape, le professeur exerce alors un rôle de modérateur. Par la suite, il assumera la phase finale avec toutes les corrections nécessaires. Au fil des semaines, la question de la terminologie se déplacera vers des difficultés moins apparentes de prime abord telles que, par exemple, le registre de langue. Le commentaire d'une étudiante étaye notre hypothèse:

L'autoévaluation m'a paru une expérience nouvelle mais très positive. Elle me permet de généraliser une problématique donnée, de mieux analyser les difficultés et d'essayer de formuler de quelle difficulté il s'agit réellement. Cela veut dire que je réfléchis toujours avant de traduire et pourquoi je traduis ainsi. Aussi, je me sens plus à l'aise dans d'autres textes quand des difficultés semblables surgissent. (Franziska G. 22-1-2001)

Cet exercice prépare l'étape suivante, en l'occurrence le questionnaire d'autoévaluation (Lee-Jahnke 1998:158-159) (voir ici l'annexe II). Ce questionnaire, conçu dans le dessein d'améliorer la faculté traduisante, met en application chez les étudiants les mécanismes du processus cognitif ${ }^{9}$ et il a porté ses fruits pendant de nombreuses années. Un questionnaire accompagne les textes à traduire et, sur la base de ce questionnaire, les étudiants sont priés de préciser pourquoi ils ont effectué telle correction ou retenu telle solution. Les améliorations obtenues étaient si frappantes dès le début de l'expérience qu'elles nous ont incité à persévérer dans cette voie ${ }^{10}$. En effet, les résultats montrent nettement les interférences et automatismes qui sont à l'origine d'erreurs. Ainsi, à titre d'illustration, citons les faux amis et les résultats d'interférences $^{11}$. Le plus souvent, on les rencontre lorsque l'opération traduisante est devenue un réflexe (Hönig 1986; Hönig et Kussmaul 1982) et à l'occasion d'automatismes, spécialement avec des étudiants avancés qui ont une approche holistique ${ }^{12}$ de la traduction (Lee-Jahnke 1998; Séguinot 1991).

Une telle approche, qui sensibilise et responsabilise les étudiants, correspond à ce qui est décrit par Alain Rosenmund (2001) dans le présent numéro, c'est-à-dire l'établissement d'un cahier des charges pour les différents textes à traduire. Puisque cette élaboration se fait avec les étudiants, ceux-ci s'exercent à mieux évaluer les points forts du texte d'après lesquels l'évaluation pourra ensuite être effectuée.

Toutes ces approches de sensibilisation tendent, on le pressent, à favoriser le processus de traduction. Il appartient au professeur, avec ses propres connaissances, d'expliquer la raison de cette manière de procéder et de devenir créatif.

Il va sans dire que les intentions généreuses qui sous-tendent toute évaluation formative sont, de prime abord, souvent méconnues par les étudiants. Car, si les résultats d'une activité d'apprentissage ne sont pas notés, certains étudiants, spéciale- 
ment en début d'apprentissage, n'investissent pas toutes leurs capacités. Voilà un point qui peut être source de difficultés.

Dans ces cas, le professeur est plutôt amené à «conduire l'activité à bon port, à maintenir le rythme, le climat, la cohérence du groupe, la continuité de l'action, le sens de l'activité, qu'à contribuer aux apprentissages prévus » (Perrenoud 1998 : 138). Dans de telles situations, un élargissement de l'intervention du professeur s'avère nécessaire: il ne peut plus se contenter de prendre en compte des facteurs de nature cognitive ou métacognitive, mais il doit considérer aussi les facteurs d'ordre sociocognitifs ${ }^{13}$ ou motivationnels. Une telle démarche peut prendre les quatre formes classiques qui sont bien connues ${ }^{14}$.

\section{b. L'évaluation formative, telle qu'elle est pratiquée dans nos cours}

D’après Prégent (1990: 53), une évaluation est formative « quand le professeur porte un jugement sur un apprentissage à n'importe quel moment pendant le processus d'apprentissage, et dans le but d'aider un étudiant à améliorer l'apprentissage en cours de réalisation».

L'idée d'évaluation formative s'est développée dans le cadre de la pédagogie de maîtrise ou d'autres formes de pédagogie différenciée, relativement peu soucieuse des contenus spécifiques des enseignements. L'accent était mis sur les remédiations, autrement dit sur une organisation individualisée de l'apprentissage, fondée sur des objectifs plus explicites, des prises d'information plus qualitatives et régulières et des interventions plus diversifiées. Aujourd'hui encore ce modèle cybernétique garde toute sa validité, à un niveau relativement élevé d'abstraction, et cela pour n'importe quelle discipline. Mentionnons pourtant un obstacle essentiel dans ce contexte: les moyens d'enseignement ne sont pas, pour la plupart, conçus pour une pédagogie différenciée assortie d'une évaluation formative (Bélair 1993). Pour aller dans ce sens, il est requis d'accepter un fort investissement dans la création ou l'adaptation d'outils didactiques. C'est ce que nous nous efforçons d'entreprendre.

Au lieu de parler de jugement de la part du professeur, nous préférons le mot aide. Cette aide peut prendre différentes formes. L'évaluation formative ne peut remplir, en effet, sa fonction primaire - celle de former - qu'à partir de quatre conditions à forte composante pédagogique:

La compétence du professeur dans sa matière;

Les aptitudes pédagogiques du professeur;

La prise en compte du point de vue des étudiants;

Le climat relationnel.

Compte tenu de ces quatre facteurs, l'évaluation formative peut être exercée sous les registres suivants:

\section{L'évaluation écrite}

Cette évaluation se pratique en règle générale sous la forme de commentaires proposés par le professeur sur les copies de traductions. Il convient d'utiliser le métalangage pour décrire les procédés «bons» ou «mauvais» que l'étudiant aura employé. Il y a lieu aussi d'en discuter les contenus pour le profit de tout le groupe. En toute hypothèse, il s'agit d'encourager les étudiants à procéder à des corrections et des 
évaluations entre eux et, en tout cas, les étudiants doivent pouvoir se sentir libres d'évaluer le travail ou la proposition du professeur. Cela conduit à une dynamique propice à la motivation et à l'apprentissage.

Comment sont ressentis ces évaluations écrites entre pairs, c'est-à-dire entre étudiants?

L'évaluation entre étudiants me paraît de grande importance, et ce, pour deux raisons. Un climat de confiance est instauré entre eux et il permet d'émettre des critiques et de recevoir celles-ci sans être blessé. Ce qui me semble la base pour un bon enseignement. Tout échange d'idées se révèle fécond dans la mesure où il permet de déceler des problèmes textuels qu'on aurait ignorés. (Maren W. 18-1-2001)

\section{L'évaluation orale}

Cette évaluation se présente surtout sous forme de traduction à vue. Il s'agit, là encore, d'encourager les étudiants à se parfaire entre eux, en commençant par les points positifs et par les solutions que d'autres ont trouvées et qu'eux-mêmes n'auraient peut-être pas repérées. Cet exercice est à l'origine de discussions fécondes. Nous avons pu constater que même les étudiants, quelque peu timides au début, finissent par trouver de l'intérêt dans cette forme d'évaluation. Car les échanges en groupe sur ces questions aident à mieux cerner et classer les difficultés rencontrées et à les pondérer lors de l'évaluation finale.

Dans la pratique répétitive de cet exercice, les étudiants sont invités à repérer les difficultés sur le résultat de leur travail et ainsi à considérer de plus en plus l'évaluation comme une partie intégrante de leur travail. En quelque sorte, un outil supplémentaire, mais indispensable, leur est fourni pour obtenir des traductions de haute qualité.

C'est lors de cet exercice que «le principe d'équité», qui veut que l'on donne à chaque élève son dû, doit être remplacé par «le principe de pertinence» qui consiste à donner à chacun ce dont il a besoin. Les passages de texte à traiter étant forcément différents les uns des autres, Perrenoud affirme dans ce contexte: «Lorsque l'on pense évaluation formative, il faut rompre avec ce schéma égalitariste. Il n'y a aucune raison de donner à tous les élèves la même dose d'évaluation formative» (1998: 142).

À la nécessité de différencier les remédiations en fonction des besoins des élèves s'ajoute aussi l'importance de soumettre les élèves à des situations d'évaluation différenciées et adaptées.

\section{c. L'évaluation sommative, un projet en cours}

D’après Prégent (1990: 50), « une évaluation est dite sommative, quand le professeur porte un jugement sur un apprentissage au terme de cet apprentissage - comme s'il faisait le bilan, la somme des connaissances qu'un étudiant a acquises pendant le cours ou une étape du cours.»

En effet, cette évaluation n'aide pas les étudiants à s'améliorer, mais plutôt à se classer. C'est un passage obligatoire dans tout enseignement. C'est un passage aussi qui suscite des craintes de la part des étudiants. Aussi nous semble-t-il souhaitable que tout enseignement soit planifié et structuré de façon à ce que l'évaluation sommative ne soit pas perçue comme une épée de Damoclès, mais comme un « défi » 
à relever par l'étudiant. Toute épreuve se présente ainsi comme une occasion de mettre en valeur les connaissances et les capacités des étudiants.

Dans son livre Pour une critique des traductions, Berman (1995: 4 $4^{\mathrm{e}}$ ) précise:

Si critique veut dire analyse rigoureuse d'une traduction, de ses traits fondamentaux, du projet qui lui a donné naissance... de la position du traducteur; si critique veut dire, fondamentalement, dégagement de la vérité d'une traduction, alors il faut dire que la critique des traductions commence à peine à exister. Si l'analyse, pour être une véritable critique, doit aussi, nécessairement, aboutir à un jugement, quelle devra être la base d'un tel jugement? Existe-t-il une base non subjective, et surtout non dogmatique, non normative, non prescriptive, une base consensuelle de jugement?

Une réponse entièrement satisfaisante à ces questions n'est pas encore trouvée, mais des recherches spécifiques ne manquent pas. Vinay (1996: 147) s'est très justement penché sur la notion d'erreur ${ }^{15}$ dans son étude en faveur d'un code de correction. Il suggère deux façons de procéder:

Les fautes doivent être expliquées de façon claire, si possible à l'aide d'un code de correction simple et connu de tous;

Il convient de les prévoir afin d'empêcher que l'étudiant ne les commette.

Actuellement, nous appliquons les trois grands paramètres suivants pour l'évaluation sommative:

\begin{tabular}{|l|l|l|}
\hline 1. Exactitude & 2. Créativité & 3. Aspect marketing \\
\hline Contenu, sens & $\begin{array}{l}\text { Traduction raisonnée, } \\
\text { idiomatique, artistique }\end{array}$ & Skopos \\
\hline Oui ou non & Excellent à insuffisant & Oui ou non \\
\hline 6 ou 0 & 6 à 0 & 6 ou 0 \\
\hline
\end{tabular}

(La notation est représentée selon le système suisse qui veut que 6 soit la meilleure note et 0 la moins bonne).

À ces trois catégories s'ajoutent des points plus différenciés qui sont représentés par des points positifs ou négatifs pour aider à établir la note aussi objective que possible. À titre d'exemple:

1. Exactitude Faux-sens;

Contresens;

Interférences - par des faux amis, par exemple;

Ambiguïtés;

Contradictions;

Omissions;

Fausse terminologie;

Manque d'uniformité (en terminologie).

2. Créativité Spécificités culturelles;

Sociolectes;

Isotopie;

Réseau sémantique;

Registre de langue;

Collocations;

Métaphores;

Jeux de mots; 


\author{
Connotations; \\ Style; \\ Orthographe; \\ Grammaire; \\ Syntaxe. \\ 3. Skopos Public cible; \\ Visée du traducteur; \\ Normes du texte (à respecter à chaque fois). Par exemple : \\ Manuel; \\ Publicité ; \\ Rapport ; \\ Brevet.
}

En ce qui concerne le skopos, il est judicieux de faire faire parfois des traductions réellement professionnelles et d'obtenir un commentaire du donneur d'ouvrage.

En possédant tout cet éventail de points sur lesquels les traductions sont jugées il est plus aisé d'être objectif. Ces points sont additionnés et permettent de schématiser la note de manière plus adéquate. Les sigles que nous utilisons sur les copies sont toutefois peu nombreux pour ne pas troubler les étudiants (voir l'annexe III). À notre avis, cela n'est pas encore un garant de l'objectivité dans l'évaluation. Pourquoi? Parce que il nous semble que, dans toute évaluation, les chercheurs ont, selon nous, mis à profit un seul côté des possibilités. En effet, dans tout examen scientifique, il y a deux voies: l'une directe et l'autre, indirecte. Comme en jurisprudence, on plaide coupable ou non coupable. En médecine, pour poser un diagnostic, il est requis de faire un diagnostic différentiel afin d'exclure toute erreur possible. En traduction, ne pourrait-t-on pas procéder par analogie? Voilà la question qui est posée et pour laquelle une recherche est en cours. Elle consiste en l'approche: thèse antithèse - synthèse. Dans cette perspective, et dans des cas de litige comme celui cité plus haut (ou «foie» et «rein» étaient confondus), nous avons mis à profit la retraduction. Ce qui, par la preuve du contraire, en démontre clairement l'erreur, si erreur il y a. Les premiers résultats de cette étude, statistiquement significatifs, seront publiés dans le courant des prochains mois.

\title{
3. Conclusion
}

Pour ce qui est de l'évaluation de la traduction, dans un cours universitaire, le succès d'une traduction devra être mesuré au degré d'adéquation à la fonction du texte et au respect de sa finalité. On évaluera les erreurs dans le contexte plus large du message global, de la structure du texte et de l'effet sur le lecteur. Il faut pourtant reconnaître que l'objectivité dans ce genre d'évaluation ne saurait être absolue en raison de la nature subjective du concept de norme pour chaque fonction, chaque genre de texte.

La pédagogie en traduction est, en quelque sorte, le gros œuvre de l'édifice. Les finitions seront toujours laissées au gré de chacun, mais la charpente doit être solide. La pédagogie, c'est aussi le climat dans lequel on apprend, la façon d'apprendre et la façon d'enseigner, la manière d'écouter et d'entendre, bref, de mettre en valeur tout le potentiel de ceux et de celles dont nous avons la responsabilité professionnelle.

Pour tout pédagogue, l'évaluation se présente comme la mesure, le dosage équilibré du «quoi» et du «comment». Elle est un chemin délicat de concertation entre le «maître» et «l'élève» pour viser, et parfois atteindre, l'excellence. 


\section{NOTES}

1. Alfred Binet (1857-1911), psychologue français.

2. Iti groups.com world-wide query

3. La problématique a été traitée avec beaucoup de pertinence par Martin Forstner (2001).

4. En l'occurrence: foie et rein.

5. Voir à propos aussi les travaux exemplaires de Kelletat (1996), de Risku (1998), de Schäffner (2000) et de Wilss (1996).

6. Cette attention peut être auditive ou bien visuelle. C'est cette dernière qui nous intéresse. L'attention visuelle sélective doit être entraînée afin de permettre de reconnaitre, par exemple, rapidement des collocations.

7. Contrairement à l'attention sélective, on parle d'attention divisée quand on essaie de faire deux choses à la fois. La question se pose si on peut valablement faire deux choses à la fois. Cela dépend. En traduction nous lisons et nous transmettons le texte lu en une autre langue. Cela exige un exercice régulier afin d'obtenir des hautes performances. C'est justement la base des cours et séminaires pratiques qui tendent à faire éliminer les interférences durant les "processus traduisants».

8. Selon Piaget (1974: 60), ce processus joue un rôle d'autant plus important qu'on se rapproche des niveaux supérieurs et du comportement. L'autoévaluation est ainsi commune aux processus vitaux et mentaux, et ses actions ont, de plus, le grand avantage d'être directement contrôlables: c'est donc dans cette direction qu'il convient de chercher l'explication biologique des constructions cognitives.

9. Une étude interdisciplinaire, récemment menée à l'Université de Genève, en donne un aperçu intéressant (Marwinski 1998). Travail de diplôme non publié.

10. Pour plus de détails, voir notre étude (Lee-Jahnke 1998: 155-183).

11. Il convient de faire la différence entre interférences cognitives, quand le traducteur fait appel à son bagage cognitif, et interférences linguistiques, qui reposent sur les connaissances linguistiques du traducteur.

12. Voir la définition dans Terminologie de la Traduction (Delisle et al. 1999).

13. Les théories sociocognitives (Bandura 1991) nous apprennent qu'un élève s'investira d'autant plus dans une activité qu'il perçoit être en mesure d'exercer un contrôle direct sur celle-ci. On peut donc en déduire que plus tôt l'étudiant sera impliqué dans le rôle de l'activité, meilleure devrait être sa motivation.

14. A) Les activités d'apprentissage autoréglées;

B) Les activités d'évaluation autoréglées;

C) Le climat de la salle de classe;

D) Le recours à des pédagogies actives;

15. Pour les cognitivistes, l'erreur est inévitable et vue d'un processus naturel d'apprentissage, vis-à-vis duquel on doit adopter une attitude de tolérance (Germain 1993:211).

\section{RÉFÉRENCES}

Bandura, A. (1991) : «Social cognitive theory of self-regulation. Organizational Behaviour and Human Decision Processes », Dordrecht, Kluver Academic Publishers, pp. 248-287.

BÉLAIR, L. (1993): "Évaluation: comparer en toute équité», Genève, Mesure et évaluation en éducation, 13-2, p. 23-45.

Berman, A. (1995): Pour une critique des traductions: John Donne, Paris, Gallimard.

BLoom, B. (1972) : Apprendre pour maittriser, Lausanne, Payot, coll. "GRETI-Information».

Bonniol, J.-J. (1981): Déterminants et mécanismes des comportements d'évaluation d'épreuves scolaires, thèse, Université de Bordeaux II.

Delisle, J. (1979) : «Une discipline en quête d'une méthodologie», Antenne, 10-6.

-, H. Lee-Jahnke, M. C. Cormier (1999): Terminologie de la traduction, Amsterdam, John Benjamins.

(2001): «L'évaluation des traductions par l'historien», Meta, 46-2, numéro spécial «Évaluation: Paramètres, méthodes, aspects pédagogiques».

Forstner, M. (2001) : «Qualitätsstandards für die Lehre», Tübingen, Narr.

Gerzymisch-Arbogast, H. (1998): Methoden des Wissenschaftlichen Übersetzens, Tübingen, Francke.

Germain, C. (1993): Évolution de l'enseignement des langues: 5000 ans d'histoire, Paris, CLE International. 
Guignard, F. (1998): "L'auto-évaluation ", L'évaluation des élèves. De la fabrication de l'excellence à la régulation des apprentissages. Entre deux logiques (P. Perrenoud, dir.), Bruxelles, De Boeck Université.

HöNIG, H. (1986) : «Übersetzen zwischen Reflex und Reflexion ein Modell der übersetzungsrelevanten Textanalyse», Übersetzungswissenschaft. Eine Neuorientierung (M. Snell-Hornby, hgg.), Tübingen, Francke.

— und P. Kussmaul (1982): Strategie der Übersetzung. Ein Lehr-und Arbeitsbuch, Tübingen, Narr.

James, W. (1890): Principles of Psychology, New York, Holt.

Kelletat, A. (1996): Übersetzerische Kompetenz. Beiträge zur universitären Übersetzerausbildung in Deutschland und Skandinavien, Frankfurt am Main, Lang.

Lachmann, R. and J. L., and E. C. Butterfield (1979): Cognitive Psychology and Information Processing, Hillsdale, Lawrence Erlbaum Associates.

Lee-JAhnke, H. (1998): "La psycholinguistique dans l'enseignement de la traduction", L'enseignement de la traduction et la traduction dans l'enseignement (J. Delisle et $\mathrm{H}$. LeeJahnke, dir.), Ottawa, Presses de l'Université d'Ottawa, coll. «Regards sur la traduction».

- (2001): Translationsqualität - Schweizer Perspektiven, Tübingen, Narr.

Levine, M. W. and J. M. Schefner (1981): Fundamentals of Sensation and Perception, London, Addison-Wesley.

Marwinski, D. (1998) : «Übertragungsstrategien und -Fehler bei Anfängern und fortgeschrittenen Lernern », Diplomarbeit, Universität Genf.

Noizet, G. et J.-P. Caverni (1978): Psychologie de l'évaluation scolaire, Paris, Presses Universitaires de France.

Perrenoud, P. (1984): La fabrication de l'excellence scolaire: du curriculum aux pratiques d'évaluation, Genève, Droz.

- (1998): L'évaluation des élèves. De la fabrication de l'excellence à la régulation des apprentissages. Entre deux logiques, Bruxelles, De Boeck Université.

Piaget, J. (1974): La psychologie de l'intelligence, Paris, Armand Colin.

Piattelli-Palmarini, M. (1979): Théories du langage, théories de l'apprentissage, Paris, Seuil.

Prégent, R. (1990): La préparation d'un cours, Montréal, École polytechnique.

Risku, H. (1998): Translatorische Kompetenz: Kognitive Grundlagen des Übersetzens als Expertentätigkeit, Tübingen, Stauffenburg.

Rosenmund, A. (2001): «Kontruktive Evaluation: Versuch eines Evaluationskonzepts für den Unterricht», Meta, 46-2, numéro spécial "Évaluation: Paramètres, méthodes, aspects pédagogiques».

Schäffner, C. (2000): Developing Translation Competence, Amsterdam, John Benjamins.

Sснміdт, S. (1988) : «Kreativität - aus der Beobachterperspektive», Kreativität - ein verbrauchter Begriff (Hans Ulrich Gumbrecht, hgg.), München.

Sснмітт, P.-A. (2001): Evaluierungskriterien für Fachübersetzungsklausuren, Tübingen, Narr.

SÉGuinot, C. (1991): "A Study of Student Translation Strategies», Empirical Research in Translation and Intercultural Studies, Savolinna, selected papers of the TRANSIF seminar.

Translator, The (2000), 6-2, numéro spécial «Evaluation and Translation» (Carol Maier, guest ed.), Manchester, St. Jerome.

VINAY, J.-P. (1996): «Enseignement de la traduction: plaidoyer en faveur d'un code de correction ", Terminology, LSP and Translation (Harold Somers, ed.), Amsterdam, John Benjamins, pp. 143-159.

Waddington, C. (2001) : «Different Methods of Evaluating Student Translations: The Question of Validity», Meta, 46-2, numéro spécial «Évaluation: Paramètres, méthodes, aspects pédagogiques».

Williams, M. (2001): «The Application of Argumentation Theory of Translation Quality Assessment», Meta, 46-2, numéro spécial "Évaluation: Paramètres, méthodes, aspects pédagogiques».

Wilss, W. (1996): Knowledge and Skills in Translator Behaviour, Amsterdam, John Benjamins. 


\section{ANNEXES \\ 1. Enquête}

Une enquête internationale via Internet a été effectuée en début de cette année. Les deux questions auxquelles il fallait répondre étaient les suivantes:

1. Qu'est-ce que vous examinez lorsque vous évaluez une traduction?

Les omissions;

Les contresens;

La grammaire;

L'orthographe;

La syntaxe;

Le style;

Les répétitions;

Les faux amis.

2. Quelles sont les capacités et aptitudes que vous attendez d'un excellent traducteur?

Connaître les nouvelles technologies;

Connaitre à fond la langue de départ;

Connaître à fond la langue d'arrivée;

Pouvoir adapter le sens aux faits de la langue cible;

Être maître de la terminologie et capable de créer ou adapter des néologismes;

Connaître l'objectif du texte à traduire ainsi que le public cible;

S'intéresser à la recherche et l'appliquer lors de traductions de sujets moins connus.

\section{Questionnaire d'autoévaluation}

\section{A. Première lecture: nature des difficultés}

1. Difficultés d'ordre lexical

a) Terminologie

b) Registre

2. Difficultés liées à la documentation

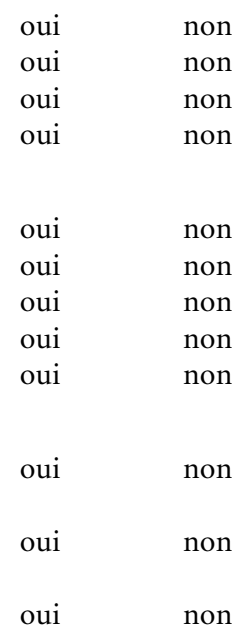

B. Deuxième lecture: source des erreurs

1. Difficultés de compréhension du TD

2. Difficultés de reformulation

a) Lexique de la langue générale

b) Syntaxe

c) Terminologie spécialisée

non

non

non

non

non

non

non

non

non

\section{Stratégie employée pour résoudre les difficultés}

1. Achievement strategy

(usage de dictionnaires ou de paraphrases)

2. Default strategy

(remémoration d'une solution fonctionnelle)

3. Reduction strategy

non

(solution de compromis insatisfaisante ou omission consciente du passage problématique - non-traduction) 


\section{Grilles}

Abréviations de correction

\begin{tabular}{|l|l|}
\multicolumn{1}{l|}{ Fautes nombre total } \\
\hline GR & \\
\hline ST \\
\hline CS \\
\hline FS \\
\hline FA \\
\hline
\end{tabular}

Bonnes solutions nombre total
\begin{tabular}{|l|}
\hline St++ \\
\hline Étoffement \\
\hline Ambiguïté intentionnée \\
\hline Solution idiomatique \\
\hline Économie \\
\hline Spécificité culturelle \\
\hline
\end{tabular}

Il est vrai que des commentaires détaillés complètent ces sigles plutôt schématiques. 